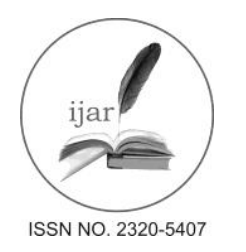

Journal homepage:http://www.journalijar.com
Journal DOI:10.21474/IJAR01

RESEARCH ARTICLE

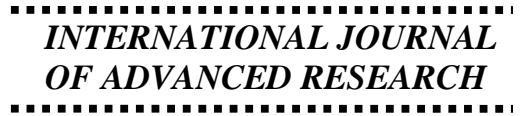

(10)

\title{
ENHANCED ENERGY EFFICIENT CLUSTERING PROTOCOL FOR WIRELESS SENSOR NETWORK.
}

1. Asst. Prof, SJBIT, Bangalore.

\author{
Rajesh L ${ }^{1}$, Niveditha H.M ${ }^{2}$, Dr. Mohan H.S .
}

2. M.Tech 4th SEM CNE, Student at SJBIT, Bangalore.

3. HOD Department of ISE, SJBIT, Bangalore.

\section{Manuscript Info}

Manuscript History:

Received: 17 March 2016

Final Accepted: 19 April 2016

Published Online: May 2016

Key words:

Energy hole, CHS Algorithm,

Dissipation, Mobile Sink.

*Corresponding Author

Rajesh L.

\section{Abstract}

Energy hole problem is one of the main problem in Wireless Sensor network. Sensor nodes near the sink acts as a relays because they are forwarding their data as well as sensors are far away from sink. Hence Energy depletion is faster in convey nodes. Suppose all the relay nodes die, far sensors can't communicate to the sink node even though they have Energy and data. This is due to Energy holes. To overcome this problem Mobile sink is used. Finding the path of mobile sink is a hard task. In this paper Mobile sinkbased Adaptive Clustering Protocol [MSIEEP] is enhanced to improve the network lifetime. Sink will be reenergized for every 10 rounds.

Copy Right, IJAR, 2016. All rights reserved.

\section{Introduction:-}

Wireless Sensor Network is made of minute devices called as Sensor nodes. The conditions like heat, Pressure, Vibration etc. are measured and monitored by Sensor nodes [1]. These monitored and measured information are then forwarded towards a static sink. Direct transmission to sink is not a good way of communication. Because it leads to unbalanced energy load distribution. So clustering Protocols are mainly designed for WSNs to improve data aggregation mechanism, Balance distribution of the energy load among sensor node. [3-6].It will increase the lifetime of Network. The clustering protocols are widely vary on nodes deployment, the network and radio models and network architecture. Suppose the sink is static sensors around that sink are acts as convey nodes, so they deplete their energy quickly resulting energy holes. This problem [7-12] leads to an early disconnection of the network so sink is isolated from the other nodes because death of its neighboring nodes. Hence mobile sink is normally accepted as an effective way to remove this problem. The network lifetime is also increases because its avoid the excessive transmission overhead at nodes that are close to the sink. Mobile sink Based Adaptive Immune Energy-Efficient clustering protocol is used to overcome the Energy hole problem.

\section{Literature Survey:-}

Many clustering Protocols for typical WSNs, which is consists of sensor nodes and static sink. Low-Energy Adaptive Clustering Hierarchy [LEACH] is the first clustering Protocol [4]. The LEACH Protocol is randomly select the cluster head that is the drawback of this protocol. Genetic Algorithm based LEACH [LEACH-GA] is proposed [5], drawback of this protocol is, During Cluster Head Selection Process residual energy of the each node is not considered. The Intelligent Agent-based Routing [IAR] is proposed to develop the efficient data delivery [8]. In this Protocol chooses some sensors as agents. Sink moves near an agent and receives data, if it is in the range of agent. It is not in the range of the Agent it does not receives the data. The Mobile Sink based Routing Protocol [MSRP] and Mobile Sink Improved Energy-Efficient PEGASIS-Based routing protocol [MIEEPB] in these protocols [10] mobile sink always follows some path and stops at some fixed temporary locations. Sensors near the fixed temporary locations dissipate 
their energy faster than other nodes. So we need to Control the movement of the mobile sink. MSIEEP Protocol during cluster head selection process distance between the member node and Cluster Head is measured. This process is done in randomly. So, we need to increase the cluster Head Selection Proposes. In turn we are going to enhance the MSIEEP Protocol.

\section{Protocol Design:-}

The protocol operation is divided into 3 phases [21],

1. Initial Phase.

2. Intermediate Phase.

3. Steady-State phase.

Initial Phase: The sink initializes the network by defining the number of nodes, the data packet size, control packet size, the size of sensing field. The connectivity between nodes and sink is always it has to be satisfied. Sink node send a request to the sensor nodes regarding ID, position Energy levels. Sensor nodes in turn send those information.

Intermediate Phase: Sink goes to the Centre of the sensing field. Cluster Head is selected usingCluster Head Selection [CHS] Algorithm. Once the Cluster Head is selected sink assigns the member nodes for each cluster. Cluster head create TDMA schedule to avoid intra-cluster collisions and CH selects a unique CDMA code to avoid inter-cluster collisions.

Set-up Phase: The sensed data is aggregated is send to sink node if sink visits all the cluster process is end. Suppose all the nodes in the region die the process will stop.

\section{Cluster Head Selection Algorithm:-}

Step 1: Initially divide the region into an equal number of nodes.

Step 2: The Normal nodes location and mobile sinklocation on coordinate axis is find out.

Step 3: The distance between the all the Normal nodes and Sink node is calculated using Euclidian Distance formula $\mathrm{d}=\operatorname{sqrt}[((\mathrm{x} 2-\mathrm{x} 1) 2+(\mathrm{y} 2-\mathrm{y} 1) 2$.

Step 4: Find minimum distance. Initially all the nodes having the Energy. So Energy is not considered for finding the $\mathrm{CH}$.

Step 5: After packet transfer Energy of the sensor nodes will be different so we consider Both Energy and Distance for finding the Cluster Head.

In this protocol, the sink is never stop at any particular locations. Its keep on moving in the Clusters so sensors around the sink changes over time. So it gives the chance to all sensors in the network to acts as covey nodes. So energy will be balanced.

\section{Implementation Details:-}

The temporary locations of the Sink node are based on minimum Energy dissipation. The cluster which is having minimum Energy dissipation in that Cluster Sink is placed. Initially Sink node is at the Centre of the Sensing field. After Packet transfer Sink find out the cluster having minimum Energy dissipation. For every 10 rounds of communication sink is reenergise. Since location of the Sink keep on changing it gives all the sensor nodes to act as relay nodes. Load balancing is also achieved.

\section{Advantages of Mobile Sink:-}

The location of Sink is changes over a time, so malicious hacker hard to know its location and damage it. So it is used in Military applications and Intrusion detection in battlefield [2]. Mobile Sink improves the network lifetime and packet drop rate. It conserve energy because data is transmitted over few hops. The number of dropped packet is also less because mobile sink closer to the sensor nodes in the sensing field. It also eliminates the Energy holes and also balance the load of data among sensors. [14][20] 


\section{Results and Discussion:-}
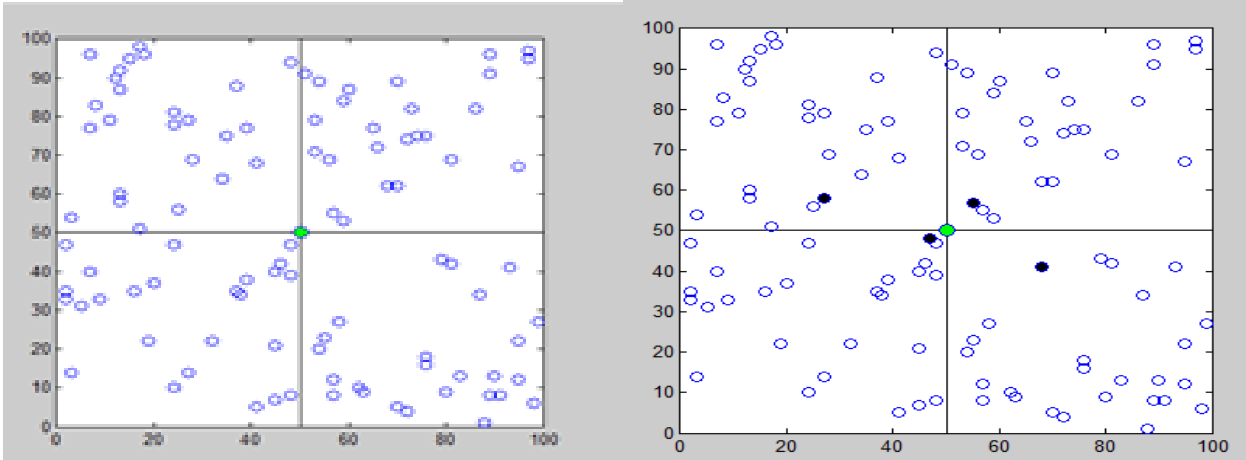

Figure 1: Initially Sink is at the Centre of the Sensing Figure 2: Cluster Heads are selected. Field.

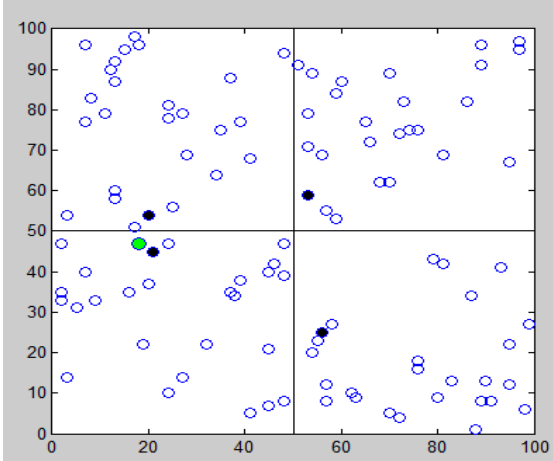

Figure 3: Sink is in the first Cluster.

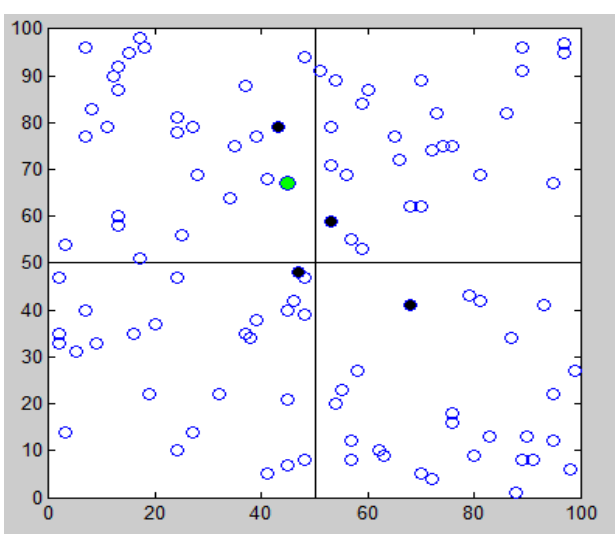

Figure 5: Sink is in the third Cluster.

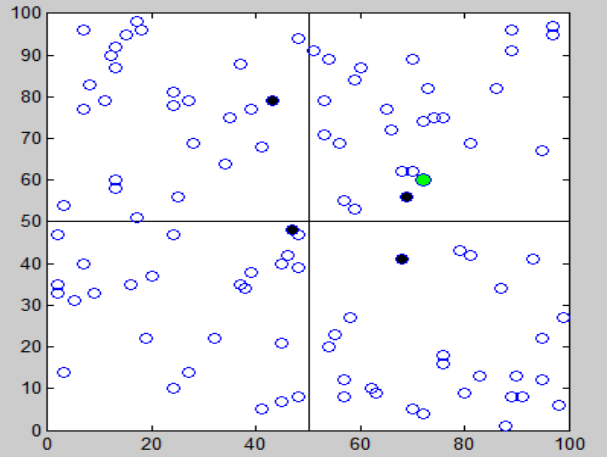

Figure 4: Sink is in the fourth Cluster.

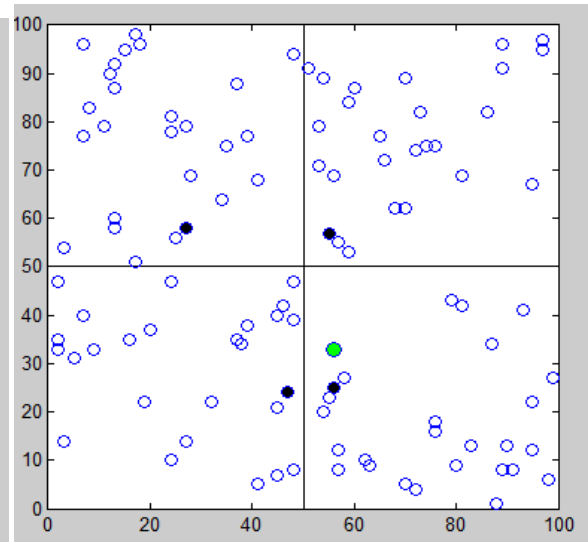

Figure 6: Sink is in the Second Cluster. 


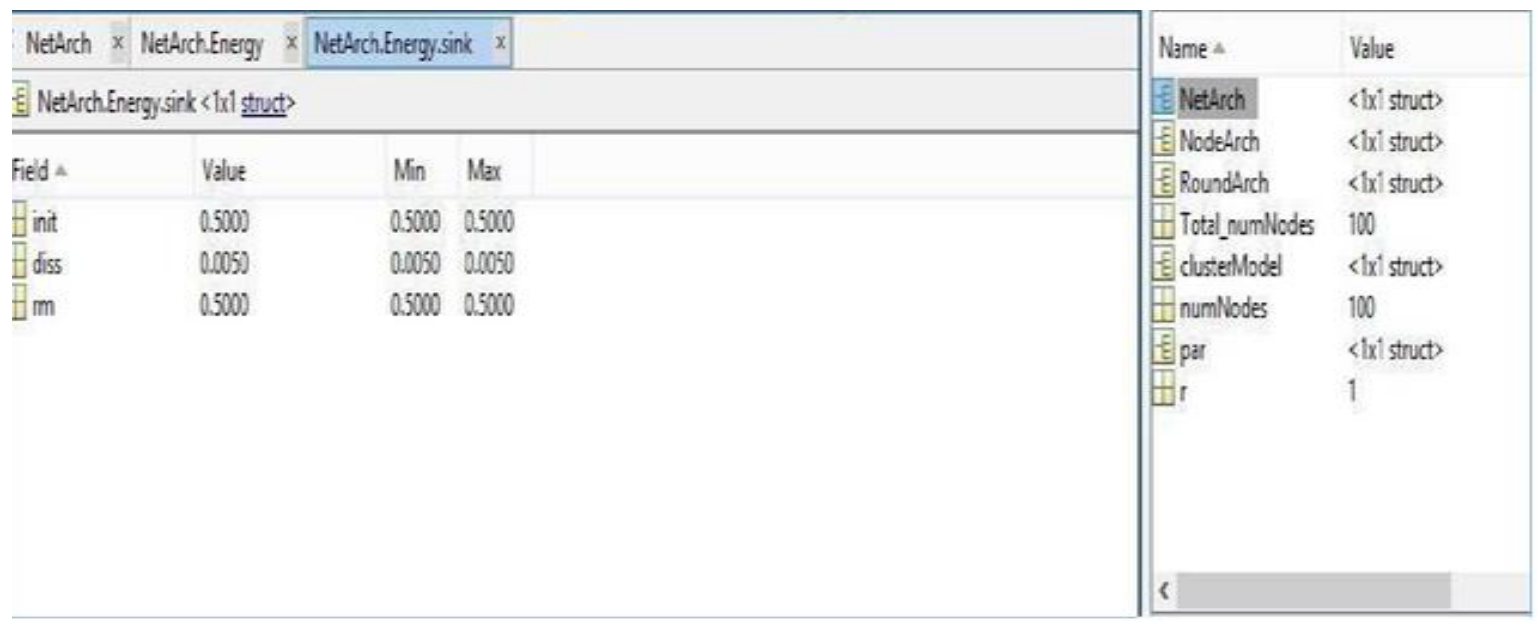

Figure 7: Sink Initial Energy.

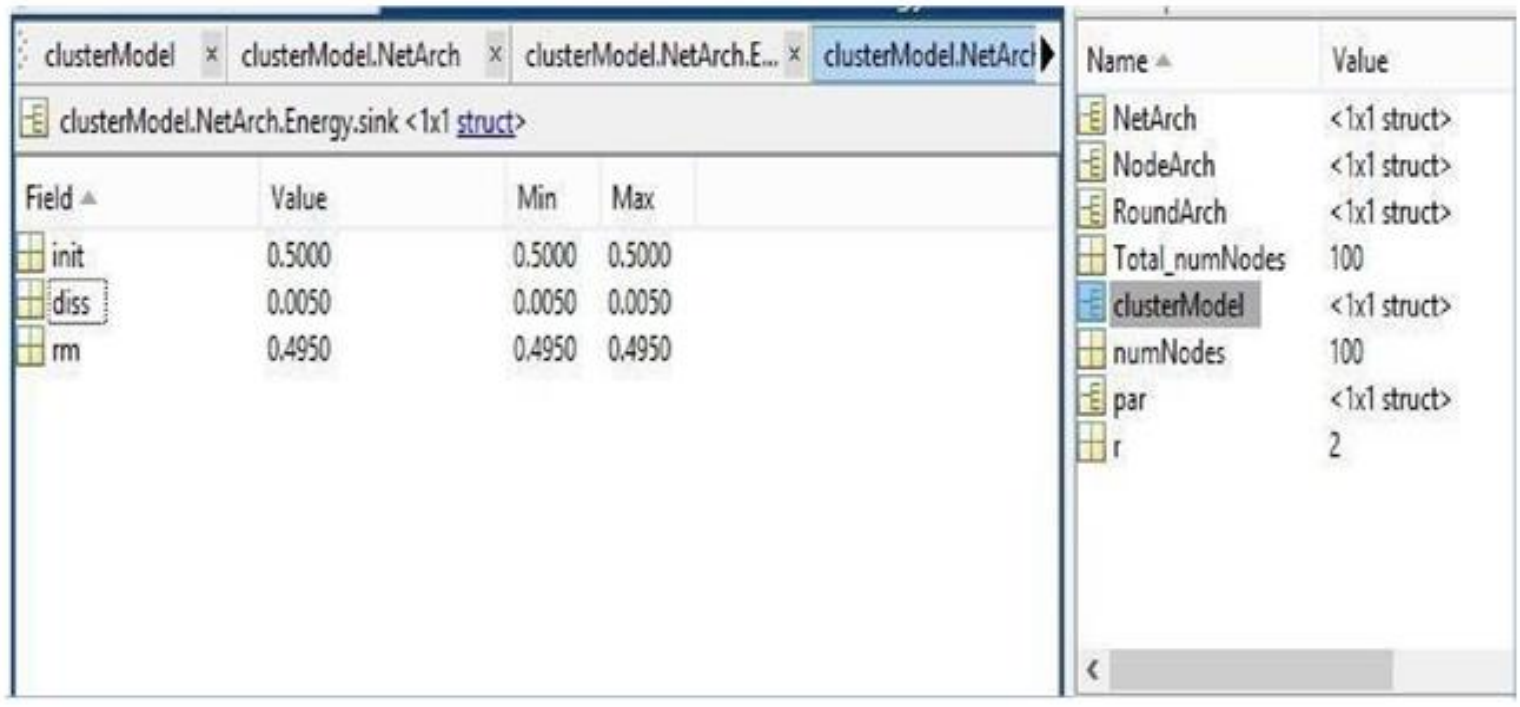

Figure 8: Sink Energy after first rounds.

\begin{tabular}{|c|c|c|c|c|c|}
\hline clusterModel $x$ & clusterModel.NetArch $x$ & clusterl & Model.NetArch.E... × clusterModel.NetArch.E... & Name - & Value \\
\hline \multicolumn{4}{|c|}{ - F clusterModel.NetArch.Energy.sink < $1 \times 1$ struct $\rangle$} & -E NetArch & $\langle 1 \times 1$ struct $\rangle$ \\
\hline Field - & Value & Min & Max & $\begin{array}{l}\text { IE NodeArch } \\
\text { FE RoundArch }\end{array}$ & $\begin{array}{l}\langle|x| \text { struct }\rangle \\
\langle 1 \mathrm{x}| \text { struct }\rangle\end{array}$ \\
\hline M init & 0.5000 & 0.5000 & 0.5000 & 円 Total_numNodes & 100 \\
\hline 五 diss & 0.0050 & 0.0050 & 0.0050 & - ElusterModel & $\langle 1 \mathrm{x} 1$ struct $\rangle$ \\
\hline$\boxplus r m$ & 0.5000 & 0.5000 & 0.5000 & $\begin{array}{l}\boxplus \text { numNodes } \\
\text { E par } \\
\mathrm{r}\end{array}$ & $\begin{array}{l}100 \\
<1 \mathrm{x} 1 \text { struct }> \\
11\end{array}$ \\
\hline
\end{tabular}

Figure 9: Sink Energy after 10 rounds. 


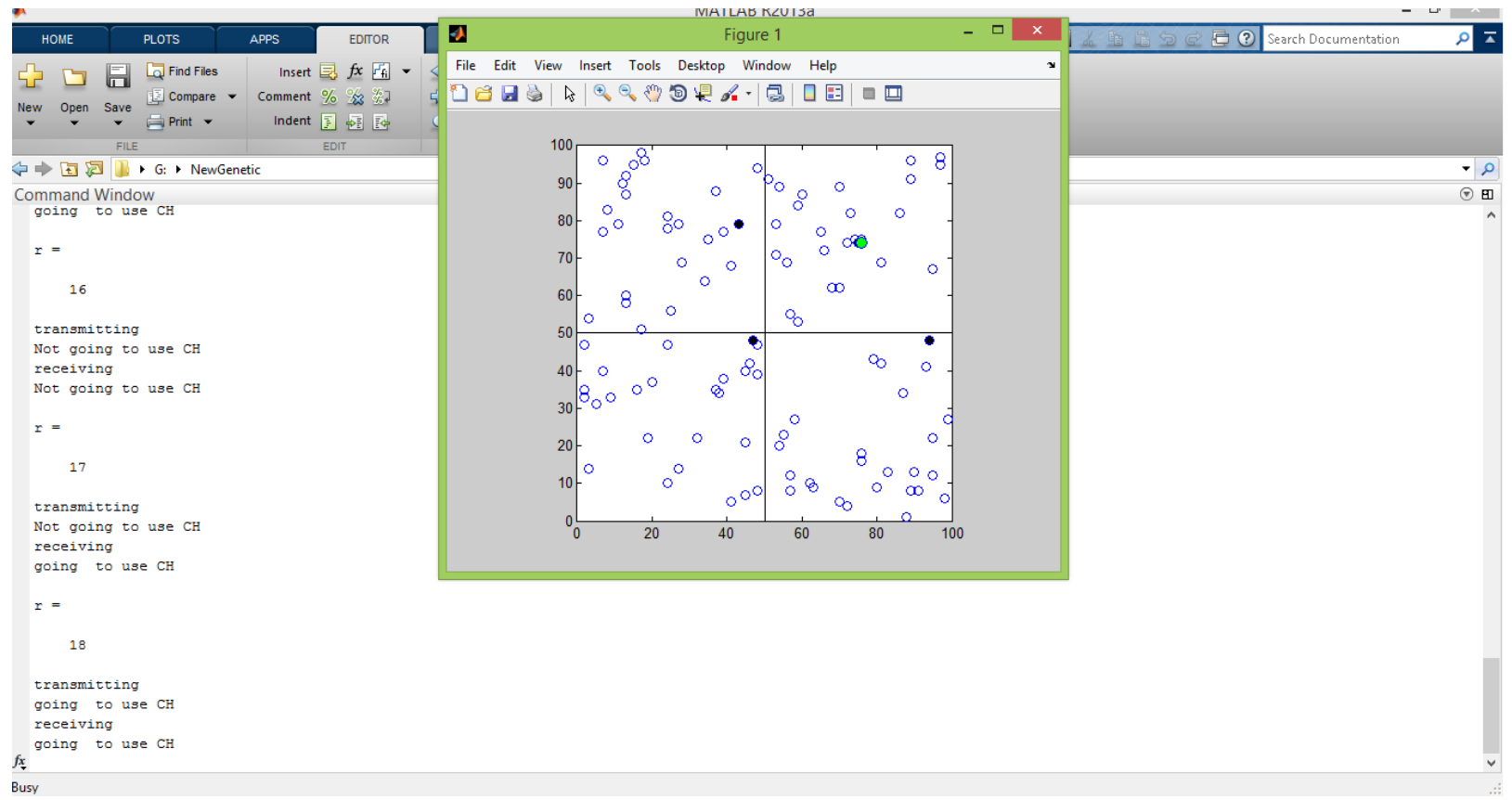

Figure 10: Figure shows that, Sensor nodes which having less distance and energy not using the $\mathrm{CH}$ during transmitting and receiving data and those are not near the Sink they use $\mathrm{CH}$ during transmitting and receiving data.

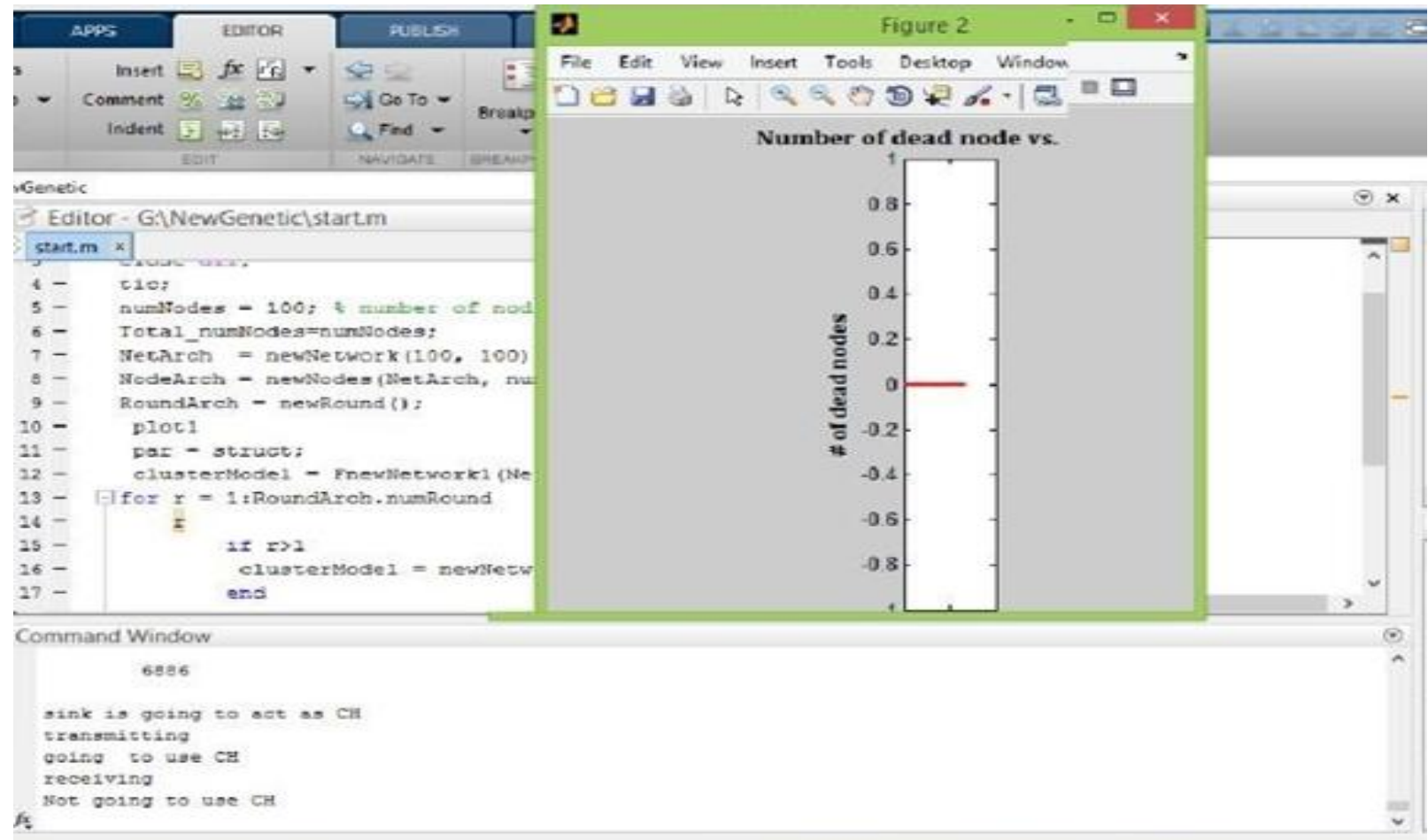

Figure 11: Sink is going to act as $\mathrm{CH}$.

Initially Sink is in the Centre of the Sensing field as shown in Figure1. After packet transferring, Cluster Heads are selected Figure 2. If fewer CHs in sensing field Energy efficiency will be high, so here for each cluster only one Cluster Head is selected. Sink find out that, which cluster is having less energy dissipation it will move to that cluster. Figure 3 shows sink is at the first cluster, Figure 4 shows sink is at the fourth cluster, Figure 5 shows that sink is in the third Cluster, Figure 6 shows that sink is in Second Cluster. So like that sink is keep on moving 
throughout the sensing field. So we can Control the movement of mobile sink, Sink is energized for every 10 rounds of communication. Suppose Sensors having less distance they can send their data to sink node and when $\mathrm{CH}$ depletes its whole energy Sink is behaves as $\mathrm{CH}$ as shown in Figure 11. $\mathrm{CH}$ are going to change for every round so each node gets a chance to become $\mathrm{CH}$ from all these aspects energy is saved inter network lifetime is increased.

\section{Performance Measures.}

In this section we compare the various clustering protocols like LEACH, PEGASIS, HEED, ACO, MSIEEP, and ENHANCED MSIEEP. Except MSIEEP protocol all the protocols have 0.5 joules initial energy and sensing field is $100 * 100 \mathrm{~cm}$. MSIEEP has 0.2 joules initial energy and sensing field range is $200 * 200 \mathrm{~cm}$. Here we are comparing the MSIEEP protocol parameters only in 4 clusters. We compare First node dead [FND], Half node dead [HND], Last node dead [LND] of various Clustering Protocols.

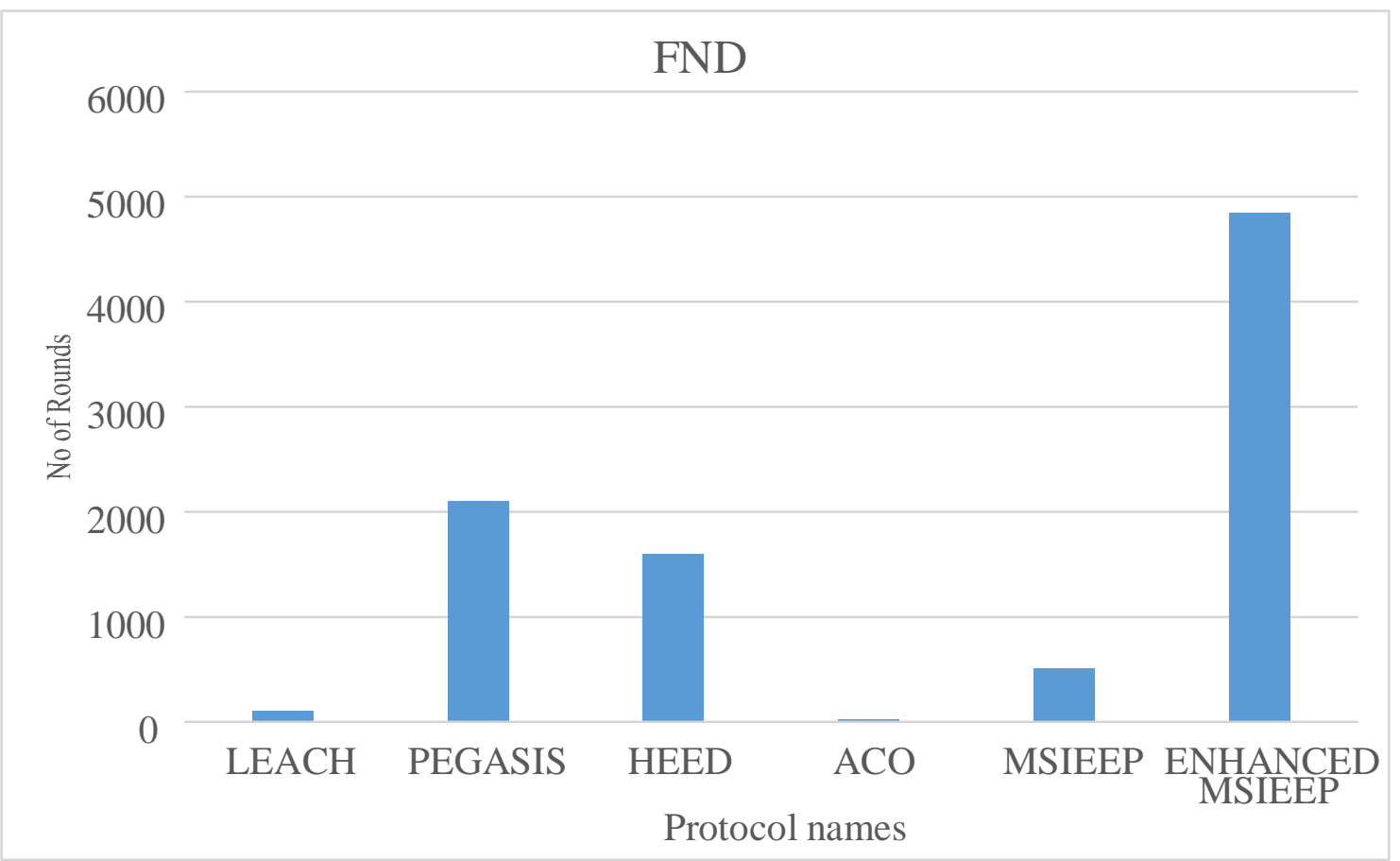

Figure 12: Comparison of FND of various Clustering Protocol. 


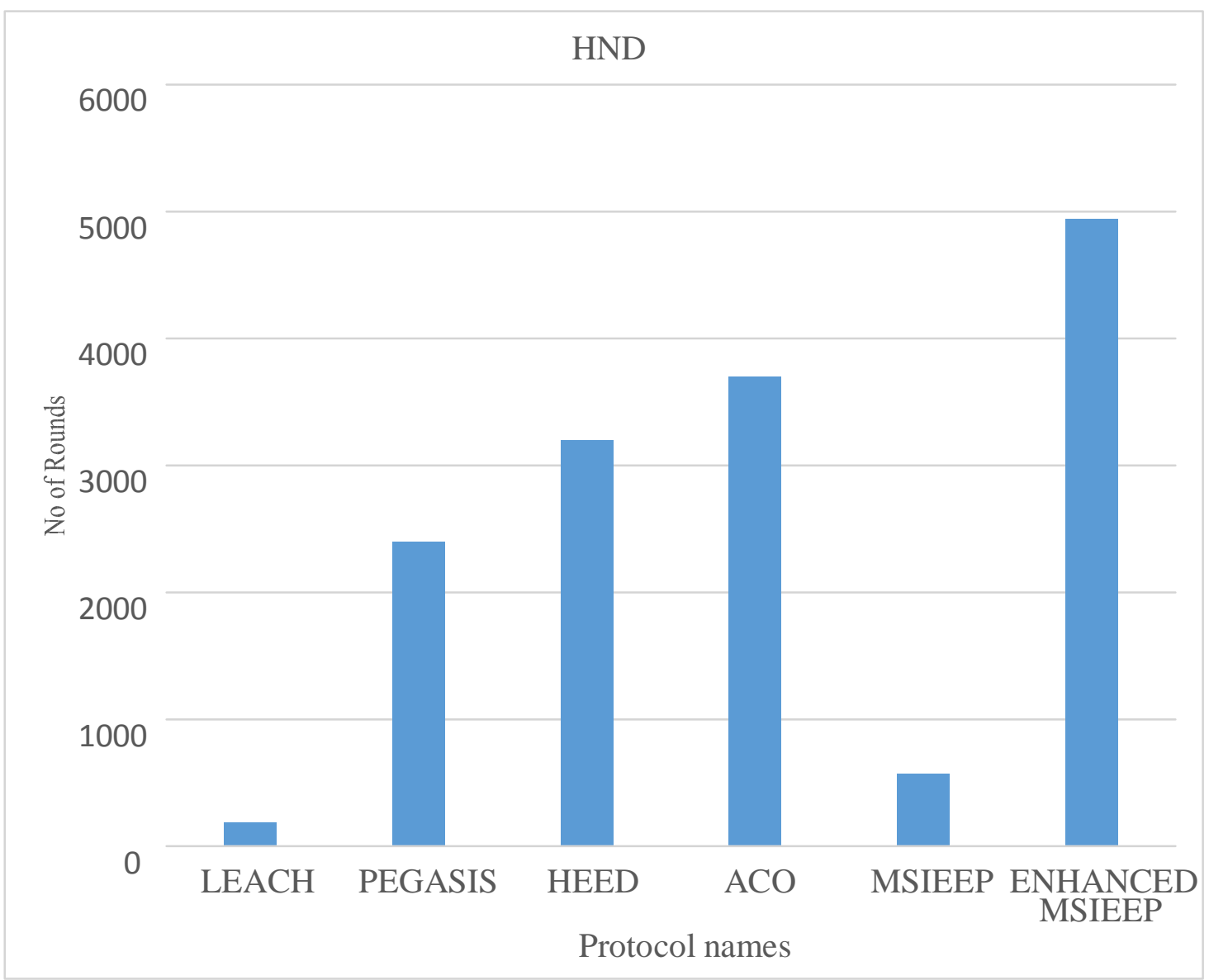

Figure 13: Comparison of HND of various Cluster Protocols. 


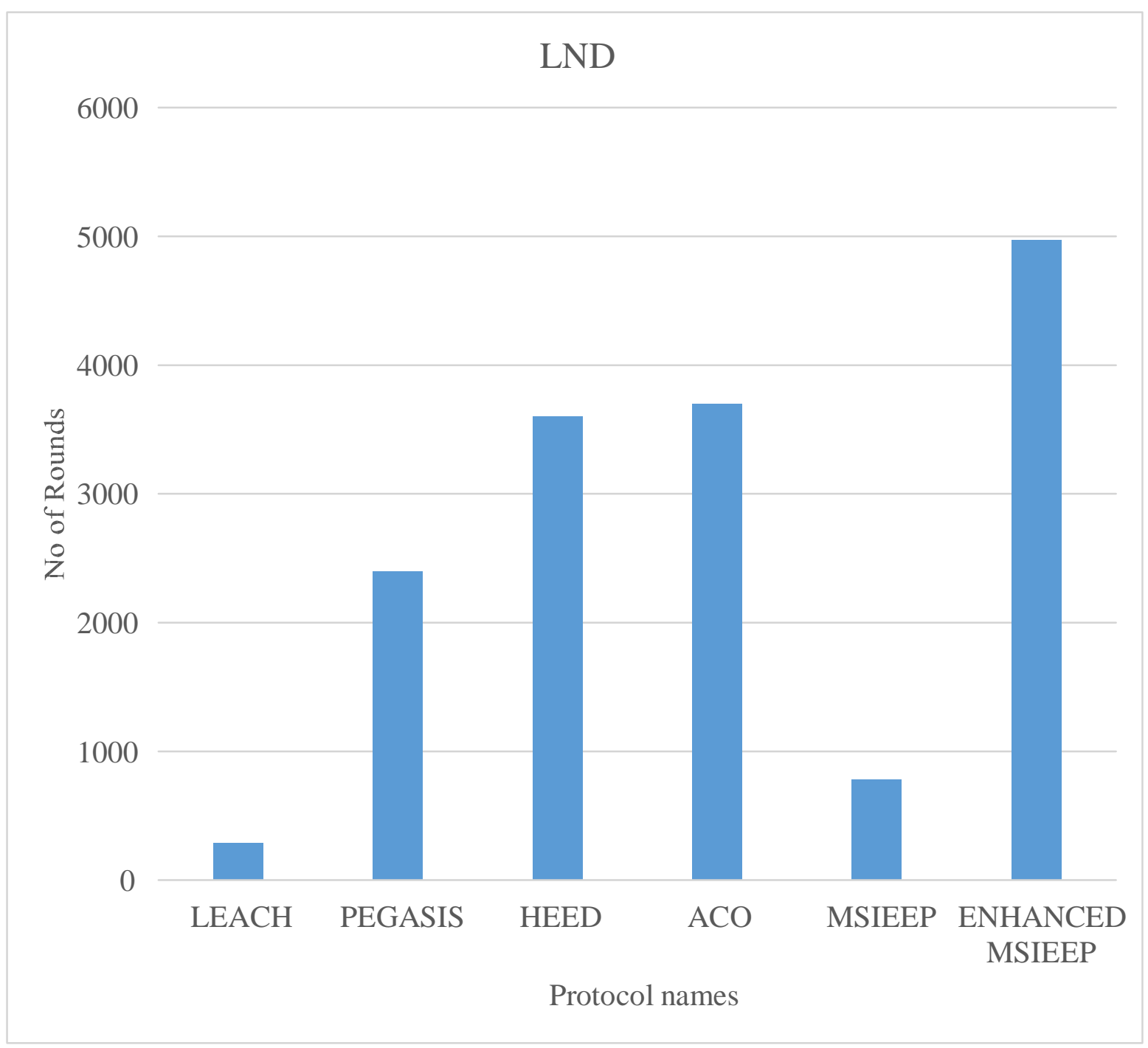

Figure 13: Comparison of LND of various Cluster Protocols.

\section{Conclusion:-}

In this paper we are enhanced the MSIEEP protocol, we are using CHS Algorithm for finding the Cluster Heads $[\mathrm{CHs}]$.During Cluster Head Selection Process We measure the distance between each and every mobile node and Sink node. Previously distance is measured randomly they are not consider each and every node. This protocol is more reliable and we can balance the Energy load among the nodes because of the sink locations changes over a time. Suppose the node which is closer to sink it can directly communicate with the sink node so it will save Energy. For every 10 rounds of communication Sink is reenergized. When Cluster head is in Sleep State or deplete its full energy Sink only acts like $\mathrm{CH}$, it collects the data from sensor nodes and aggregated. So energy is saved intern Network lifetime is increased. We also compare the various Clustering Protocols except MSIEEP all the protocols have $0.5 \mathrm{~J}$ energy. MSIEEP has $0.2 \mathrm{~J}$ Energy, the parameters like FND, HND, and LND are compared. Figure 11, 12, 13 shows the comparison results. Here we consider MSIEEP in only 4 clusters, from these results we can show the differences between MSIEEP and ENHANCED MSIEEP. From all these aspects we enhanced the MSIEEP Protocol up to some extent. 


\section{References:-}

1. S. K. Singh, M. P. Singh, and D. K. Singh, "Routing protocols in wirelesssensor networks-A survey," Int. J. Comput. Sci. Eng. Surv., vol. 1, no. 2,pp. 63-83, Nov. 2010.

2. E. B. Hamada and G. Chelius, "Strategies for data dissemination tomobile sinks in wireless sensor networks," IEEE Wireless Commun.,vol. 15, no. 6, pp. 31-37, Dec. 2008.

3. A. A. Abbasi and M. Younis, "A survey on clustering algorithms forwireless sensor networks," Comput. Common., vol. 30, nos. 14-15,pp. 2826-2841, Oct. 2007.

4. W. B. Heinzelman, A. P. Chandrakasan, and H. Balakrishnan, "An application-specific protocol architecture for wireless microsensornetworks,” IEEE Trans. Wireless Commun., vol. 1, no. 4, pp. 660-670,Oct. 2002.

5. J.-L. Liu and C. V. Ravi Shankar, "LEACH-GA: Genetic algorithm-based energy-efficient adaptive clustering protocol for wireless sensor networks," Int. J. Mach. Learn. Comput., vol. 1, no. 1, pp. 79-85,

6. Apr. 2011.

7. K. G. Vijayvargiya and V. Srivastava, "An amend implementation on LEACH protocol based on energy hierarchy," Int. J. Current Eng.Technol., vol. 2, no. 4, pp. 427-431, Dec. 2012.

8. S. Basagni, A. Carosi, E. Melachrinoudis, C. Petrioli, and Z. M. Wang, "Controlled sink mobility for prolonging wireless sensor networks lifetime," Wireless Newt., vol. 14, no. 6, pp. 831-858, Dec. 2008.

9. J.-W. Kim, J.-S. In, K. Hurl, J.-W. Kim, and D.-S. Edom, "An intelligent agent-based routing structure for mobile sinks in WSNs,” IEEE Trans.Consum. Electron., vol. 56, no. 4, pp. 2310-2316, Nov. 2010.

10. W. Liang, J. Luo, and $\mathrm{X}$. Xu, "Prolonging network lifetime via controlled mobile sink in wireless sensor networks," in Proc. IEEEGLOBECOM, Miami, FL, USA, Dec. 2010, pp. 1-6.

11. B. Nazir and H. Hasbullah, "Mobile sink based routing protocol (MSRP)for prolonging network lifetime in clustered wireless sensor network, "inProc. ICCAIE, Kuala Lumpur, Malaysia, Dec. 2010, pp. 624-629.

12. S. Mottaghi and M. R. Zahabi, "Optimizing LEACH clustering algorithm with mobile sink and rendezvous nodes," AEU-Int. J. Electron.Commun., vol. 69, no. 2, pp. 507-514, Feb. 2014.

13. M. R. Jafri, N. Javari, A. Javari, and Z. A. Khan, "Maximizing the lifetime of multi-chain PEGASIS using sink mobility,” World Appl.Sci. J., vol. 21, no. 9, pp. 1283-1289, Mar. 2013.

14. Lu Yuting, Wang," Energy Hole Solution Algorithm in Wireless Sensor Network", JOURNAL OF NETWORKS, VOL. 9, NO. 4, APRIL 2014.

15. Yongyi Liu, Siqing Yang, Huiyong Yuan," Avoiding Energy-hole in Wireless Sensor Networks with Hybrid Communication Model", 2011 International Conference on Computer Science and Information Technology (ICCSIT 2011), IPCSIT vol. 51 (2012) @) (2012) IACSIT Press, Singapore DOI: 10.7763/IPCSIT.2012.V51.38

16. Ruthvic S D, Ravi B, Udaya Kumar Shenoy," Energy Optimization using Neighbourhood based Weighted Rendezvous Technique for Wireless Sensor Networks', International Journal of Computer Applications (0975 - 8887) Volume 120 - No.8, June 2015.

17. Apostolos Iisa demertzis Demertzis, Konstantinos Oikonomou, "Avoiding Energy Holes in Wireless Sensor Networks with Non-Uniform Energy Distribution", Published IN: IEEE Transactions on Parallel and Distributed Systems (Volume:19, Issue: 5 ).

18. X. Lu, Y. Ding, and K. Hao, "Immune clonal selection algorithm fortarget coverage of wireless sensor networks,” Int. J. Model., Identification., Control, vol. 12, nos. 1-2, pp. 119-124, Jan. 2011.

19. M. Abo-Zahhad, S. M. Ahmed, N. Sabor, and A. F. Al-Ajlouni, "A new method for fastening the convergence of immune algorithms using an adaptive mutation approach,” J. Signal Inf. Process., vol. 3, no. 1,pp. 86-91, Feb. 2012.

20. K. Shinghal, A. Noor, N. Srivastava, and R. Singh, "Power measurements of wireless sensor network node," Int. J. Comput. Eng.Sci., vol. 1, no. 1, pp. 8-13, 2011.

21. Mohammed Abo -Zahra, Senior Member, Sabah M,Ahmed,Nabil Sabor ,"Mobile Sink-Based Adaptive Immune Energy-Efficient Clustering protocol for Improving the Lifetime and Stability Period of Wireless Sensor Networks",IEEE sensor Journals, vol.15 NO 8,August 2015.

22. M. M. Sandhu, M. Akbar, M. Behzad, N. Javaid, Z. A. Khan, andU. Qasim, "Mobility model for WBANs," in Proc. 9th Int. Conf.BWCCA, Guangzhou, China, Nov. 2014, pp. 155-160 